\title{
On the Convergence of Wired and Wireless Access Network Architectures
}

\author{
Dr. William H. Lehr ${ }^{1}$ and Dr. John M. Chapin ${ }^{2}$ \\ Massachusetts Institute of Technology
}

\begin{abstract}
Wired and wireless access networks continue to evolve toward higher-capacity, multi-service systems. Recent wireless broadband networks such as 3G LTE and WiMax provide a general-purpose IP platform with over-the-top services at the application layer, which is similar to the design of wired IP platform networks. This paper examines whether wired and wireless access networks are likely to converge on a common architecture, or if not, whether wireless networks are likely to converge on a common wireless architecture. We conclude that the answer to both questions is No. We identify fundamental and persistent differences between wired and wireless networking that will propel wired and wireless access network architectures on divergent evolutionary paths. Whereas we expect wired broadband access networks to continue to evolve toward a common general-purpose platform architecture, we expect wireless networks to remain heterogeneous. The inherent scarcity of radio frequency spectrum emerges as the key reason for this prediction. We examine the implications of divergent evolutionary paths for market structure and regulatory policy.
\end{abstract}

\section{Introduction}

The co-evolution of the wired and wireless Internet offers significant potential for the creation and transformation of markets for communications services and products. One critical question facing stakeholders in this process is whether the architecture and capabilities of wired and wireless access networks will eventually converge. The degree of convergence will

\footnotetext{
1'wlehr@mit.edu

${ }^{2}$ jchapin@mit.edu
} 
determine whether wired and wireless services function as substitutes or complements, with strong implications for market structure and regulatory policy.

Wired access networks that were historically distinct, notably switched voice versus broadcast video, are evolving to a common "platform network" architecture. Wired platform networks use the high capacity of fiber-optic-rich physical networks and the general-purpose capability of IP-based protocols to support a triple play of voice, video, and data services. In the wireless domain, albeit with a lag, there is a similar trend towards increased capacity and towards providing a range of services over a common IP-centric network infrastructure.

These trends point to an apparent future convergence on a platform network architecture for both wired and wireless access networks. In this paper, we identify fundamental and persistent differences between wired and wireless networks that make such convergence unlikely. The primary factor is the limited availability of radio frequency spectrum, which imposes severe capacity constraints on wireless networks. The capacity-efficiencies associated with specialized wireless architectures offset the scale, scope, and learning economies that are pushing wired networking toward a general-purpose platform architecture.

Given an understanding of the fundamental differences between wired and wireless access networks, we analyze the economic and policy implications. The analysis presented here is partial because we focus on the implications of technical and architectural issues. We recognize that the evolution of markets, industry structure, and public policy will depend on many other factors that are beyond the scope of this paper. Other factors include organizational aspects of operator businesses, what service options are successful in the marketplace, and the market response to changing regulatory policies. 
The balance of this paper is organized into four sections. In Section 2, we provide a highlevel analysis of the most important persistent differences between wired and wireless networking. In Section 3, we offer our forecast for how these differences will affect the future evolution of wired and wireless access networks. In Section 4, we address some of the likely implications of our forecast for regulatory policy and market structure. Section 5 summarizes our conclusions and suggests directions for future work. For convenience, we refer simply to wired and wireless networks; it should be understood that all discussion in this paper concerns access networks that provide last-hop connectivity to a large number of end users.

\section{Wired versus Wireless Networking}

Table 1 summarizes the key features that underlie persistent differences in wired and wireless networking. We consider each in turn in the following sub-sections.

\begin{tabular}{|l|c|c|}
\hline \multicolumn{3}{|c|}{ Table 1 Persistent Key Differences in Wired v. Wireless Networking } \\
\hline & Wired & Wireless \\
\hline Capacity & Abundant & Scarce \\
\hline Topology & Point-to-point & Broadcast \\
\hline Reliability & Reliable & Unreliable \\
\hline Mobility & Fixed & Mobile \\
\hline
\end{tabular}

\subsection{Capacity}

Wired networks have an inherent capacity advantage over wireless networks because of the significantly greater frequency range that may be carried by wired infrastructure. This is a simple matter of physics. For example, a single coaxial cable has a useful frequency range on 
order of $1 \mathrm{GHz}$ while a single optical fiber has a useful frequency range of over $1,000 \mathrm{GHz} .^{3}$ The entire wireless $\mathrm{RF}$ spectrum $(3 \mathrm{~Hz}$ to $300 \mathrm{GHz})$ fits easily in a single fiber. With multi-fiber bundles, each cable replicates many times more than the RF spectral capacity.

The comparison of raw frequency range availability is a good first-order indicator of the data communications capacity potential of different technologies. To make the discussion complete, two second-order effects need to be mentioned. First, the maximum data rate depends not only on frequency range but also on the encoding or modulation scheme that is used. Encoding and modulation techniques for wireless communications continue to improve over time, increasing the effective capacity for a fixed frequency range. Second, data communications capacity depends on how the frequencies are allocated and utilized. Studies of RF usage have found that most of the desirable RF spectrum is not actively carrying transmissions most of the time in most places. ${ }^{4}$ This suggests that there is substantial room for the RF spectrum to be shared more intensively. We believe strongly (and have argued elsewhere) that such sharing is both desirable and necessary for the healthy evolution of wireless technology and services. ${ }^{5}$ However, neither of these second-order effects - the potential for improved modulation schemes and for improved sharing - is sufficient to overcome the massive frequency range disadvantage that limits the capacity of wireless networks to far less than the capacity of wired networks. The significance of the capacity limitation is shown in part by the high prices paid in spectrum auctions in recent years.

${ }^{3}$ See Goleniewski (2007).

${ }^{4}$ A number of studies have measured RF spectrum occupancy. For example, in a series of studies conducted by Shared Spectrum Company for the US National Science Foundation in 2004 and 2005 (http://www.sharedspectrum.com/measurements/), data showed that the average occupancy over all locations tested was 5.2\% and that the maximum occupancy was in New York City at $13.1 \%$.

${ }^{5}$ See Chapin and Lehr (2007). 
Each of the other persistent differences we discuss below has as at least one of its primary effects an additional burden on wireless capacity. Thus we view capacity limits as the most important fundamental difference between wired and wireless networking. A common theme emerges: the fundamental scarcity of wireless capacity increases the opportunity cost of spectral inefficiencies, thereby providing stronger incentives in wireless than in wired networks for using service-specific technologies at the lower layers of the network architecture.

\subsection{Topology}

Wired networks are inherently point-to-point. The cables carrying the communications signal focus the transmitted energy to specific devices at either end of the link.

Wireless is inherently a broadcast technology, in the sense that transmitted energy is spread over a geographic area in which there are likely to be multiple receiving devices. As a result, interference is a major issue for wireless networks. Interference can be generally understood as the adverse outcome that occurs when signal energy is delivered where you do not want it. Interference imposes further capacity constraints on wireless networking. The communications resources allocated to a wireless system (frequencies, time slots, codes) cannot be fully used by the transmissions between any pair of devices. ${ }^{6}$

On the other hand, services that require delivering the same information to multiple receivers can be made substantially more efficient if the inherent broadcast nature of wireless is exploited properly. This creates a strong incentive to provide specialized support for broadcast services. In wired networks, this incentive also exists but is substantially reduced because

\footnotetext{
${ }^{6}$ There are techniques for focusing transmitted RF energy that in the limit may approximate the point-topoint delivery performance of wired media. Doing this at frequencies below $3 \mathrm{GHz}$ requires phased array antennas that are too large to build into handsets and currently too expensive to deploy widely in fixed stations. Above $3 \mathrm{GHz}$ the antenna problem is significantly easier, but propagation effects make such frequencies uneconomical to use for access networks intended to provide broad coverage.
} 
capacity is generally so abundant. Even popular content such as live sporting events is largely distributed over point-to-point data transmissions to each user if accessed as streaming video over the web. Some physical layer technologies (e.g. passive optical distribution networks) support broadcast but this is a secondary operation mode not widely used in commercial systems.

The topology differences have systematic implications for wired and wireless networking that go beyond capacity constraints. Topology differences affect the balance of costs between initial deployment and later scaling of a service, which in turn drive different market structures (see Section 3.2).

\subsection{Reliability}

Raw data transmission in wired networks is generally much more reliable than in wireless networks, in three ways. First, at the transmit power and propagation distances appropriate for a commercially viable system, a wired network has orders of magnitude fewer bit errors than a wireless network. Second, wired links offer near 100\% uptime except during equipment failures, whereas wireless links have occasional dropouts (periods when the receiver gets no data) due to propagation variations caused by device mobility or a changing environment. ${ }^{7}$ And third, wired networks are highly predictable and can be engineered to provide the exact service desired, whereas the coverage and performance of a wireless network is difficult to predict or adjust precisely.

Methods of compensating for all these forms of wireless network unreliability consume additional capacity. The overheads are significant. For example, in the most-widely used mobile

\footnotetext{
${ }^{7}$ Dropouts can be prevented by increased transmit power. However other factors such as the need to minimize interference and to minimize mobile device battery weight push for system designs with low link power margins.
} 
voice technology - GSM full-rate-43\% of the capacity allocated to each voice call is used for error correction (9.8 kbps out of $22.8 \mathrm{kbps})$.

Specializing the reliability compensation mechanisms to the application service can reduce overheads and therefore significantly increase overall system capacity. For example, key frames of compressed video can be sent with greater redundancy than incremental update frames (whose loss causes fewer visible artifacts to the end user). Voice sample retransmissions can be suppressed if the tight latency deadline imposed by a full duplex voice conversation has expired. SMS messages can be resent at a later time rather than immediately since end users do not expect fixed time deadline delivery.

Specializing the reliability compensation mechanisms in ways like this requires that the lowest layers of the network design be adapted to the service being provided to the user. Thus the inherent unreliability of wireless transmission combined with the high opportunity cost of spectral inefficiency creates strong incentives to make wireless networks service-specific. This effect is not present in wired networks.

\subsection{Mobility}

Wired networks provide a fixed service, whereas the core value of wireless networks is mobility in all its forms. Wireless networks support communications while in motion. Wireless networks provide ubiquitous coverage, while a wired network only provides service where there is a plug. Wireless networks offer flexibility in choosing and changing service location, while wired networks require cable rerouting to change location.

Provisioning for mobility further limits the capacity of wireless networks compared to wired networks. Communications resources (spectrum, power, time slots, codes) need to be reserved in the area where the receiver might be. Optimizations that reduce resource usage 
become harder to implement when the communications channel is changing or unpredictable. Size and weight of mobile devices becomes a driving design goal, limiting battery capacity and antenna capability, which in turn limits transmit power and hence capacity.

Mobility effects can be exploited to increase wireless network capacity. In some cases this can be done without knowledge of the application service. ${ }^{8}$ However, the most widely used techniques depend on optimizing lower layers for expected characteristics of the application service. For example, CDMA 1xEVDO and similar data-oriented systems exploit the latency tolerance of Internet data services and channel variations due to mobility when making channel allocation decisions. ${ }^{9}$ This is another example of capacity-driven pressure to make wireless networks service-specific.

The inherent orientation of wireless services towards mobility also leads to a different role for the end user in network provisioning and management than occurs in wired networks, with implications for industry market structure (see Section 4.4).

\section{Divergent Evolutionary Paths}

The fundamental differences analyzed in the previous section result in divergent evolutionary paths for wired and wireless networking.

\subsection{Technical evolution of wired networks}

Historically, the wired networks deployed by legacy telephone and cable television providers had physical and technical architectures specialized to the service they offered. As these so-called "silo" networks are replaced by fiber-rich networks capable of supporting a

\footnotetext{
${ }^{8}$ See Grossglauer and Tse, 2002.

${ }^{9}$ See Bhushan et al. (2006).
} 
triple-play of voice, video, and data, a common network architecture has emerged that we call the "platform network" (Figure 1).

A platform network is built from general purpose media, transmission technology, and networking mechanisms. The various services (voice, video, data) are supported as application level data streams on top of this general purpose data communications platform. Although media and lower-layer transmission technology vary from network to network, all current commercial deployments use the Internet Protocol suite for higher layer transmission and networking purposes. $^{10}$

While the evolution of wired networks to platform architectures is far from complete, it is well advanced in urban areas. For example, as of March, 2009, fiber-to-the-home was available in North America to approximately 15.2 million homes (13\% penetration), with 2.7 million homes subscribing to a triple play of voice, video, and internet access, largely from Verizon. ${ }^{11}$ As of June, 2009, 6.8 million homes in the US subscribed to a similar triple play from Comcast via last-mile coaxial cable. ${ }^{12}$

Wired networks are converging to the general-purpose platform architecture because of strong improvements in scale, scope, and learning economies. These combine to reduce network equipment, design, and management costs. Moreover, a general purpose platform architecture facilitates the flexible deployment of new services and allows network capacity to be quickly reallocated as demand shifts across services or users. These provide substantial operating cost

${ }^{10}$ See Lehr \& Chapin (2009a) for a more precise definition and further discussion of the evolution of broadband platform architectures.

${ }^{11}$ Fiber To The Home Council, "North American Market Update, April 2009." Retrieved July 6, 2009, from http://www.ftthcouncil.org/sites/default/files/RVA.FTTH_Apr09.060109.pdf.

${ }^{12}$ Comcast Corporation, “Comcast Launches New Worldwide Calling Plan.” Press release, June 25, 2009. Retrieved July 6, 2009, from http://www.comcast.com/About/PressRelease/Press/ReleaseDetail.ashx?PRID=884.

Page 9 of 28 
benefits for service providers and access to a wide range of third-party over-the-top services for end users.

\subsection{Inefficiencies of platform networks}

The benefits of platform networks come at a cost in technical efficiency. Efficiency suffers in part because of the overheads required to share a common network among multiple applications. Some mechanism is necessary to link each chunk of data to the information required to route, deliver, and process the information correctly. In Internet Protocol networks, this function is performed by the IP header that labels each packet of data. While IP header overhead is small for most services, it becomes significant for services that require frequent transmission of short packets, notably two-way voice calls. For example, a GSM radio channel that is optimized for voice can carry eight voice streams, whereas this same radio channel (same channel width, same modulation) can only handle three voice streams if they are carried as Voice-over-IP. ${ }^{13}$

Another source of inefficiency is the inability to apply application-specific optimizations at the lower layers of the network. Such optimizations include physical layer broadcast for broadcast services, error protection and recovery mechanisms tuned to the specific needs of different applications, and resource allocation mechanisms that exploit the latency tolerance of data oriented applications. An integrated "cross layer" approach to resource management

${ }^{13}$ The channel described is the standard $200 \mathrm{kHz}$ wide 8-slot GSM channel. GSM voice streams are coded at $13 \mathrm{kbps}$ or less before forward error correction. In VOIP, header overhead approximately doubles the data rate per voice stream before error correction. Other effects add further overhead, particularly differences in error correction design required to support general IP traffic, leading to more than twice the resource consumption per stream. 
provides significant capacity benefits that are not available in a general-purpose platform architecture. $^{14}$

A final type of inefficiency arises from design decisions made in the Internet Protocol suite. These performance costs are not fundamental to all possible platform networks but are deeply rooted in commercial practice. The traditional IP suite provides only "best effort" packet delivery in the underlying network platform. In best effort delivery, the network provides no guarantees against packet loss or delivery delays. Resource management to balance offered load to available capacity, and recovery from packet loss, are handled by a separate higher layer of the system called the Transport Control Protocol (TCP). This design has proven immensely valuable for the Internet, enabling it to integrate a wide range of network technologies that have different loss and delay characteristics.

For non-real-time services such as file transfer, best effort delivery plus TCP is effective and efficient. However, the real-time requirements of newer multimedia services such as Voiceover-IP and streaming video have challenged the traditional Internet best effort model. The most common way to work around this in commercial IP access networks is to provision excess capacity. With enough excess capacity and sufficiently high data rates, the packet losses and delivery delays of best effort service are reduced sufficiently to satisfy the requirements of multimedia applications. Over-provisioning corresponds to a direct reduction in efficiency. While there are multiple approaches to improve multimedia support (known as Quality of Service or QoS) in the Internet, over-provisioning remains a critical element in IP access networks. $^{15}$

\footnotetext{
${ }^{14}$ See Lehr and Crowcroft (2005), Kawadia (2004), or Shakkottai, Rappaport and Karlsson (2003).

${ }^{15}$ See Odlyzko (2000) for discussion of over-provisioning in data networks.
} 
The technical inefficiencies associated with the platform network architecture have been acceptable in wired networks because of the extremely high capacity and low error rates of modern fiber optic transmission technology. This has made it possible to adopt the architecture for its operational and end-user benefits. The tradeoffs are different in wireless networks operating under stringent capacity limits.

\subsection{Technical evolution of wireless networks}

Wireless networks today are still largely organized technically as silo networks. The dominant systems used for voice telephony worldwide (GSM, UMTS voice-mode, and CDMA2000) use connection-oriented transmission and switching technology. The emerging systems for video distribution (MediaFLO in the USA, DVB-H elsewhere) use broadcastspecific transmission technology. The current generation of mobile Third Generation (3G) wireless systems supporting Internet data services (UMTS HSPA and CDMA 1xEVDO) are specialized for web browsing and file transfer applications. The technical differences between

these silo networks are largely hidden from consumers. Access to what are in fact multiple wireless networks is bundled into a single integrated mobile customer device.

The wide deployment of wireless 3G networks supporting IP may suggest that wireless platform architectures are taking off, leading to a convergence of wired and wireless network architectures. However, this is an incorrect interpretation. No service provider has announced plans to provide its mass-market voice or video broadcast services as an over-the-top application over its IP platform. The wireless IP networks are optimized for Internet data access and are simply too inefficient for the reasons described in the previous section to replace the other wireless silo networks. 
It is highly unlikely that deployment of fourth generation networks will change this fundamental dynamic. The 4G system currently expected to dominate, Long Term Evolution ("LTE"), is an IP-centric data network with impressive capabilities and a platform network architecture. ${ }^{16}$ While LTE offers substantial improvements in spectral efficiency relative to current $3 \mathrm{G}$ systems, it also enables increased per-user capabilities that are expected to contribute to demand growth. A careful study of the balance between user demand growth and technology improvements concluded that meeting user demand would require an additional 500 to 1000 $\mathrm{MHz}$ of commercial spectrum in the USA by 2020 , all below $5 \mathrm{GHz} \cdot{ }^{17}$ Given that government (public safety, defense, etc.) see their need for spectrum increasing, and given the challenges of clearing previously allocated spectrum, it is unreasonable to expect that this demand will be fully met through new allocations. Hence future $4 \mathrm{G}$ systems will be at least as capacity limited as current wireless networks, so the technical inefficiencies associated with running all services over the top of a common platform will continue to be economically unacceptable.

Commercial providers apparently anticipate this outcome. For example, there has been substantial recent effort to integrate "voice fallback" capability more tightly into the LTE standard, enabling providers to couple a dedicated voice network (perhaps a new design more spectrally efficient than GSM) with their LTE data network. ${ }^{18}$

Consequently, we predict a future in which wireless networks remain technically heterogeneous. A mix of specialized and general-purpose facilities-based networks will co-exist as part of the wireless landscape. While this may sound like a simple continuation of the current

\footnotetext{
${ }^{16}$ Bogineni et al. (2009a, 2009b).

${ }^{17}$ See ITU (2006).

${ }^{18}$ See Donegan (2009).
}

Page 13 of 28 
model, we anticipate significant changes coming in technical approach and economic structures. ${ }^{19}$ For the current discussion, it is sufficient to observe that the future of wireless services is multiple heterogeneous networks rather than convergence to a shared platform network for all services as is occurring in wired networks.

\subsection{Market structure}

The competitive environment is and will remain distinctly different for wired and wireless networks, due to opposing entry and capacity expansion economics.

In the entry phase, when a network is first deployed, wired networks have very high upfront costs, whereas wireless networks permit more incremental investment (setting aside the costs of securing spectrum access rights). The high up-front costs for wired networks are driven by their fundamental point-to-point topology. Cables need to pass every home or location that might be served by the network. Deployment of wired infrastructure also requires securing access to rights-of-way and conduits or outside structures such as telephone poles. In contrast, wireless services can exploit their broadcast topology to reduce up-front costs. Service can be initially offered in a region with a relatively small number of base stations, each transmitting at high power to cover a large area. Capital investment to provision each customer can also be small, since with proper technical design the inherent mobility of wireless devices enables endusers to self-install and make local adjustments to improve service. ${ }^{20}$ As market penetration and

\footnotetext{
${ }^{19}$ See Lehr and Chapin (2009b) for a more detailed discussion of our forecast of what the wireless future will look like.

${ }^{20}$ Examples of local adjustments include TV antenna alignment and nomadic foraging for better cell phone signal quality. Not all wireless access networks permit self-installs or end-user adjustments. Counterexamples include satellite and some fixed wireless broadband services in which antenna placement is highly sensitive. Such systems have higher up-front capital investment requirements associated with creating a field service organization of trained installers.
} 
customer demand grows, capacity can be expanded by adding "in-fill" base stations while reducing transmit power, thereby increasing spatial reuse of available spectrum resources.

Once a network is fully built out in a region and enters its capacity expansion phase, the balance of costs shifts. In wired networks, fiber cables that include initially dark fibers allow large capacity expansion at relatively low incremental cost. For wireless networks, there is a density of base stations beyond which adding new tower sites offers diminishing returns (either due to interference problems or because real-estate costs and zoning problems increase dramatically). Capacity expansion of wireless networks beyond that point requires acquiring additional spectrum, installing smart antennas, or substantially reducing cell sizes. ${ }^{21}$ All of these options are expensive, making it costly to support significant improvements in the number of subscribers or per-subscriber services.

The differing entry and capacity expansion economics for wired and wireless networks imply differing competitive dynamics for wired and wireless service providers. For wired networks, the number of providers that can be economically viable in any local area is strictly limited. Due to the high up-front network investment, wired service providers require a relatively high take-rate of homes passed to be economically viable, usually in excess of $25-30 \%{ }^{22}$ Indeed, there are very few instances in the U.S. where there are more than two wired operators in a local area. This suggests that some areas previously enjoying duopoly facilities-based competition (DSL over copper vs cable modem over coax) may see the deployment of only one

21 This may be accomplished by installing femtocells on the customer's premises that rely on the customer's wired broadband access to backhaul mobile services used within the home.

${ }^{22}$ For example, minimum break-even take rates (subscribers as a share of homes passed) for fiber deployments are typically in excess of 30\% (see for example, BroadbandProperties (2008) or FTTH Council (2008)). 
fiber optic network. ${ }^{23}$ At the same time, the low cost of capacity expansion means that new overthe-top services can easily be accommodated in an existing platform network. Therefore a potentially important source of competitive discipline in wired services is entry by new thirdparty over-the-top service providers. Good examples are Vonage in the U.S. and Skype internationally.

For wireless networks, the incremental investment profile for new deployments means that wireless services are normally economically viable at lower take rates than would be required for wired services. ${ }^{24}$ This helps explain why many local areas in the U.S. are served by four or more wireless facilities-based providers while they only have one or two wired facilitiesbased providers. At the same time, the high cost of capacity expansion after initial deployment renders the introduction of third party over-the-top services problematic. Therefore it is more natural to look to entry by new facilities-based wireless providers for competitive discpline rather than to entry by over-the-top providers.

To summarize, in wired networking, we expect there will be a relatively small number of wired facilities-based providers in each market (e.g., at most two in most markets in the U.S.). Most wired service providers will offer a triple play bundle of services over similar broadband platform architectures. In wireless, we expect to see a larger number of facilities-based competitors offering services over a mix of heterogeneous wireless infrastructures. While some of the wireless service providers will offer general-purpose internet access services, others will run specialized networks offering niche services (e.g., as is the case with the MediaFLO video

\footnotetext{
${ }^{23}$ This seems especially likely in areas where wired duopoly is less prevalent, such as in Europe. This helps motivate the current interest in Europe in segmented geographic regulation (Pupillo \& Amendola, 2009).

${ }^{24}$ Minimal sustainable market shares for mobile operators may be single digit percentages (Sharma, 2008).
} 
distribution services offered by Qualcomm). In the wireless case, we expect that entry by new facilities-based providers will be a more potent source of competitive discipline than in the wired case.

\section{Implications for Industry Structure and Regulatory Policy}

The divergent evolutionary paths for wired and wireless broadband have important implications for industry structure and regulatory policy. In the following sub-sections, we briefly explore several of these implications.

\subsection{Technology neutral regulation}

Technology neutral regulation of services is intended to eliminate asymmetric treatment based on the underlying network technology. ${ }^{25}$ Adopting such an approach is consistent with the goal of symmetric regulation that proponents have argued will assist in promoting telecommunications competition. ${ }^{26}$ The approach is also consistent with proposals for "layered regulation" that have been advanced as a solution to convergence-driven challenges to silo-based regulation. ${ }^{27}$ The 2002 reform of the European Union's regulatory framework to focus on

${ }^{25}$ Our use is consistent with Mindel and Sicker (2006): "technologically neutral refers to policy and business decisions of a service being made without basing the decision on the underlying physical plant that delivers the service."

26 Schankerman (1996) argues in favor of symmetric regulation to promote competition in telecommunications. Technical neutrality is a form of symmetric regulation. See ECTA (2008) for a carrier-centric view in favor of technological neutrality.

${ }^{27}$ Sicker, Mindel and Cooper (1999) proposed evaluating Internet interconnection regulation based on a layered model that segmented the analysis horizontally by protocol layer. Subsequent authors went further in advocating a move toward horizontal regulation (i.e., services and applications regulated independently of underlying network services) as an alternative to legacy silo-based, vertical regulation (e.g., Frieden, 2004; Whitt, 2004; or Werbach, 2002). This in turn has spawned a number of critiques (see Marcus, 2002; or Gifford, 2004). See Sicker and Blumensaadt (2005) for a review of this debate. 
markets rather than infrastructures reflects a conscious effort to move toward technological neutrality. $^{28}$

Perhaps ironically, technology neutrality may become less relevant for the regulation of wired providers as their networks converge toward similar architectures. At the same time, as wireless networks become increasingly capable, pressure will likely increase to extend technology neutrality to wireless services. We have already seen an example of this in the ongoing "Network Neutrality" debate. ${ }^{29}$ In a paper that is consistent with the move toward technological neutrality, Tim $\mathrm{Wu}$ (2007) expanded the debate by suggesting that policymakers ought to consider how "Network Neutrality" might apply to wireless providers. In response, a number of economists have argued against this suggestion, relying principally on an assessment of wireless competition to explain their position. ${ }^{30}$ As we have explained, because wired and wireless networks differ in fundamental technical ways, and because capacity is a dominant constraint for wireless networks that is much less salient in wired networks, such an extension of technology neutrality would be bad policy.

\footnotetext{
${ }^{28}$ See Mindel and Sicker (2006), Marcus (2002), and Reding (2006).

${ }^{29}$ This is a debate over the need for regulatory rules to constrain how broadband ISPs manage traffic. It was sparked by $\mathrm{Wu}(2003)$ and has subsequently spawned a large academic and policy literature. The debate over "Network Neutrality" is related to, but distinct from the debate over technology neutral regulation which is why we retain the quotes. See, Lehr, Peha, and Wilkie (2007) for a collection of papers offering divergent perspectives on the "Network Neutrality" debate.

${ }^{30}$ See, for example, Ford, Koutsky, and Spiwak (2007), Rosston and Topper (2009), and Hahn, Litan and Singer (2007).
} 


\subsection{Interconnection}

Policymakers have long recognized that intercarrier compensation regulation, which includes rate regulation of interconnection and access charges, is in need of reform. ${ }^{31}$ One proposal to simplify regulation is "bill-and-keep" in which usage-based intercarrier interconnection payments are eliminated. Each carrier recovers the usage-based costs associated with terminating the traffic of their interconnection partners from their own subscribers.

Our technical analysis suggests that usage-based costs in wireless networks are likely to be systemically higher and more variable than is the case with wired network termination. Reasons for this include capacity limits, which increase both marginal per-minute costs and enduser prices, and the unpredictability of wireless links (Section 2.3). These effects argue against the economic efficiency of setting a single uniform rate for wireless interconnection, especially one that does not differentiate between wired and wireless termination (Gabel, 2002). Indeed, it may provide additional support for adopting bill-and-keep as the only reasonable option. ${ }^{32}$

\subsection{Broadband universal service policy}

The current interest among policymakers in promoting universal broadband service raises the question of how to define a minimal standard for broadband and how to evaluate relative needs for improved service (e.g., to target public broadband funding). ${ }^{33}$

${ }^{31}$ See Notice of Proposed Rulemaking (NPRM), in the Matter of Developing a Unified Intercarrier Compensation Regime, Before the Federal Communications Commission, CC Docket No. 01-92, April 19, 2001.

32 Atkinson and Barnekov (2000) argue that any regulated rate is likely to be inefficient and propose a bill-and-keep approach as the best way to ensure competitive neutrality.

33 The American Recovery and Reinvestment Act (ARRA) of 2009 includes \$7.2 billion to promote broadband access to unserved and underserved communities. The federal agencies responsible for disbursing these funds are not adopting separate definitions for fixed and mobile broadband and are seemingly trying to be technologically neutral with respect to the sorts of broadband projects that may be

Page 19 of 28 
We have identified fundamental differences between wired and wireless access networks which lead to different market structures. Therefore we believe that at least two definitions of broadband are needed: one for fixed (mostly wired) broadband service and one for mobile (wireless) broadband service. While they may be substitutes for some users in some markets, ${ }^{34}$ they offer distinct capabilities and this distinction will persist into the future.

More specifically, although wireless networks will continue to improve, it is likely that they will continue to lag wired services in terms of what policymakers may consider a minimal level of quality (i.e., average and peak data rates supported, reliability, etc.). An acceptable minimal standard for wired performance is likely to be too high a threshold for wireless. However, wireless offers mobility. Which is better may depend on the situation. ${ }^{35}$

\subsection{End-user empowerment and wireless competition}

Finally, it is worth highlighting an important way in which wireless competition has the potential to be systematically different from wired competition. The end-user plays a much more direct role in the network management process than in wired networking. While wired broadband customers may select among wired providers and their tiered offerings, once the service is installed, the customer has only a limited ability and opportunity to manage his or her service quality. In contrast, because of the local variability in wireless transmission performance, wireless customers are accustomed to playing a much more active role. This takes the form of

funded. A notable exception is the exclusion of satellite-based wireless services (see, for example, http://www.telecompetitor.com/first-look-at-the-broadband-stimulus-rules/).

${ }^{34}$ Ward and Woroch (2005).

${ }^{35}$ For example, a fixed service is not much use to a homeless person (see Graham (2007)).

Page 20 of 28 
physically moving to better position a cell phone's antenna to receive a stronger signal, or moving the location of the WiFi radio to improve signal coverage within the home. ${ }^{36}$

Indeed, the WiFi phenomenon has demonstrated the willingness and capability of endusers to actively deploy networking infrastructure. Moreover, the potential for mass deployment of ad hoc and/or mesh networking technology suggest that we could go much further than we have to date in exploring the limits of end-user provided networking. In contrast, it is hard to imagine mass deployment of wired infrastructure by end customers. ${ }^{37}$ This suggests that the principal mode of competition in wired is likely to remain competition among a relatively small number of facilities-based wired service providers. ${ }^{38}$ Although a similar prognosis might apply to wireless (e.g., if the challenges of deploying sustainable $4 \mathrm{G}$ mobile businesses pushes the industry toward further consolidation), there is the potential for end-user deployed infrastructure to offer a novel vector for the emergence of competition and network innovation. ${ }^{39}$

\section{Conclusions}

Technological innovation in communications has expanded the number of ways to provide any given service. Thus, software and hardware-based solutions may compete as substitutes. Fast and cheap CPUs may reduce the need for expensive transport (e.g., using compression). Wired and wireless networking may offer alternative ways to transmit broadband

${ }^{36}$ While the quality of mobile telephony has improved dramatically to render such adjustments unnecessary in many locales, this sort of real-time adjustment to improve performance remains important for more advanced media-intensive services (e.g., $3 \mathrm{G}$ broadband) and new types of wireless (e.g., RFID and sensors).

${ }^{37}$ Community-based and municipal fiber may be viewed as limited exceptions.

${ }^{38}$ A few facilities-based providers may support a much larger number of resellers.

${ }^{39}$ Additionally, we expect to see a proliferation of separate facilities-based niche wireless networks (e.g,. for RFID, for public safety, for ad hoc networking, for satellite communications, etc.) that will not be mirrored by the relatively homogeneous future we anticipate for wired networking. 
data or telephone calls. From a policy perspective, the expansion of technical options creates both a challenge and a temptation. The challenge is to identify and understand the technical differences that have significant policy and economic implications. The temptation is to conclude that the many available options have reduced the importance of technology-specific constraints, freeing policy-makers to regard communications networks as "black boxes." For example, it may be tempting to view innovation in networking technology as eroding the differences between wired and wireless networks and services. In such a view they are becoming substitutes in the technical sense, in that they use similar architectures and have similar capabilities. This would enable them to function as substitutes in the economic sense, in that they are substitutes in consumption. ${ }^{40}$

This paper has focused on the fundamental, and we believe persistent, differences between wired and wireless technologies. With respect to wired networking, we see facilitiesbased providers continuing the current trend of evolution from silos to a common platform network architecture. A diverse and flexible bundle of services will be offered over the top of these platform networks. However, wireless networks are unlikely to evolve to a similar architecture as wired networks. Indeed, we predict that wireless networks will fail to converge to a common wireless architecture. Instead wireless networks will remain heterogeneous, with many of them specialized to particular services. For example, when GSM networks are shut down starting 8-10 years from now, we predict they will be replaced by more spectrally-efficient voice-specialized networks that are tightly coupled with the provider's 4G data networks, rather than being replaced by VOIP traffic streams running over the top of those $4 \mathrm{G}$ networks.

\footnotetext{
${ }^{40}$ As we noted earlier, the extent to which services may be perceived as complements or substitutes in the market depends on more than the underlying technical architecture used to provide the services.
} 
The heterogeneity and specialization of wireless networking that we observe and predict to continue can be traced, in part, to the fundamental capacity disadvantage faced by wireless relative to wired technologies. The capacity disadvantage of wireless is reinforced by topological differences (broadcast instead of point-to-point), reliability differences (wireless is less reliable and predictable), and mobility differences (wired is fixed and wireless is mobile).

The differences between wired and wireless network technology and architecture lead to economic differences, with significant implications for public policy. For example, there will be an enduring need to craft interconnection and universal service policies and network neutrality policies that are distinct for fixed and mobile services. Additionally, we view new-entrant facilities-based providers and end-user deployed infrastructure as much more important and likely vectors for innovation and competitive discipline in wireless than in wired networks. The appropriate policy frameworks to facilitate effective competition will therefore be different in the two cases.

Looking forward, for wireless network competition and innovation of the forms we have highlighted in this paper to be fully realized, a number of policy areas must be carefully addressed. Major areas for innovation concern spectrum policy and ways of increasing spectrum sharing - but these are topics for another paper. ${ }^{41}$

${ }^{41}$ See, for example, Chapin and Lehr (2007) or Lehr and Chapin (2009b). 


\section{References}

Atkinson, Jay and Christopher Barnekov (2000), "A Competitively Neutral Approach To Network Interconnection," OPP Working Paper Series, No. 34, Federal Communications Commission, December 2000.

Bogineni, Kalyani, Reiner Ludwig, Preben Mogensen, Vish Nandlall, Vojislav Vucetic, Byung Yi, and Zoran Zvonar (eds) (2009a), "LTE Part 1: Core Network," IEEE Communications Magazine, vol. 47, no. 2, February 2009.

Bogineni, Kalyani, Reiner Ludwig, Preben Mogensen, Vish Nandlall, Vojislav Vucetic, Byung Yi, and Zoran Zvonar (eds) (2009b), "LTE Part II: Radio Access," IEEE Communications Magazine, vol. 47,. No. 4, April 2009.

BroadbandProperties (2008), "Ingredients for a Successful FTTH Overbuild," Broadband Properties Summit 2008 Coverage, June 2008, pages 58-60 (available at: http://www.bbpmag.com/2008issues/june08/BBP_June08_SummitOverbuild.pdf).

Chapin, John and William Lehr (2007), "The path to market success for dynamic spectrum access technology," IEEE Communications Magazine, May 2007.

Donegan, Michelle (2009), "New Specs Deepen LTE Voice Dilemma," Unstrung News Analysis, July 7, 2009. Available online at http://www.unstrung.com/document.asp?doc id=178915).

ECTA (2008), ECTA Review 2008, European Competitive Telecommunications Association (ECTA), 2008 (available at: http://www.ectaportal.com/en/upload/ECTA_Review_2008.zip).

Ford, George, Thomas Koutsky, and Lawrence Spiwak (2007), "Wireless Net Neutrality: From Carterfone to Cable Boxes," Phoenix Center Policy Bulletin No. 17, April 2007.

Frieden, Robert (2004), "Adjusting the horizontal and vertical in telecommunications regulation: a comparison of the traditional and a new layered approach", Federal Communication Law Journal, Vol. 55 No.207, pp.209.

FTTH Council (2008), "Municipal Fiber to the Home Deployments: Next Generation Broadband as a Municipal Utility," FTTH Council North America, April 2008 (available at: http://www.ebusinessforum.gr/engine/index.php?op=modload\&modname=Downloads\&action=d ownloadsviewfile\&ctn $=1889$ \&language $=\mathrm{el})$.

Gabel, David (2002), "Competitive Market Approach to Interconnection Payments in the US," in Networking Knowledge for Information Societies: Institutions and Intervention, eds. Robin Mansell, Rohan Samarajiva and Amy Mahan, pp. 132-140, Delft University Press, 2002. 
Gifford, Raymond (2004), "The Uses and Misuses of the Layered Model," The Skeptical Regulator, (August 2004), http://www.pff.org/irle/skepticalregulator/skepticalregulator2.3.html.

Goleniewski, Lillian (2007), Telecommunications Essentials, Second Edition, Addison-Wesley: New York, 2007.

Graham, Kevin (2007), "Wireless a lifeline for homeless," St. Petersburg Times (Hillsborough Edition), April 7, 2009 (available http://www.sptimes.com/2007/04/09/Hillsborough/Wireless_a lifeline_f.shtml).

Grossglauer, Matthias and David Tse (2002), "Mobility increases the capacity of ad hoc wireless networks," IEEE/ACM Transactions on Networking, vol. 10, no. 4 (August 2002) 477-486.

Hahn, Robert, Robert Litan, and Hal Singer (2007), "The Economics of Wireless Net Neutrality," Journal of Competition Law and Economics, 2007 3(3):399-451.

ITU (2006), "Estimated spectrum bandwidth requirements for the future development of IMT2000 and IMT-Advanced," International Telecommunications Union (ITU) ITU-R Working Party 8F Report M.2078, May 2006 (available at: http://www.itu.int/publ/R-REP-M.2078/en).

Kawadia, Vikas (2004), "Protocols and Architecture for Wireless Ad Hoc Networks," PhD Dissertation, Department of Electrical Engineering, University of Illinois, Urbana-Champaign, 2004 (adviser: P. R. Kumar)

Lehr, William, Jon Peha, and Simon Wilkie (eds) (2007), Special Section on Network Neutrality: International Journal of Communication (volume 1, 2007), August 2007 (http://ijoc.org/ojs/index.php/ijoc/issue/view/1).

Lehr, William and Jon Crowcroft (2005), "Managing Access to a Spectrum Commons," IEEE DySPAN Conference Proceedings, Baltimore, MD, November 2005.

Lehr, William and John Chapin (2009a), "Divergent Evolutionary Paths for Wired and Wireless Broadband," invited paper presented to Workshop on Wireless Technologies: Enabling Innovation \& Economic Growth, The Georgetown Center for Business and Public Policy, Washington, DC April 17, 2009 (available at: .

Lehr, William and John Chapin (2009b), "Hybrid Wireless Broadband," paper presented to the 37th Research Conference on Communication, Information and Internet Policy (TPRC), George Mason University School of Law, Arlington, VA, September 25-27, 2009.

Marcus, J. Scott (2002), "The potential relevance to the United States of the European Union's newly adopted regulatory framework for telecommunications," Office of Plans and Policy Working Paper Series \#36, Federal Communications Commission.

Mindel, Joshua and Douglas Sicker (2006), "Leveraging the EU regulatory framework to improve a layered policy model for US telecommunications markets," Telecommunications Policy 30 (2006) 136-148. 
Odlyzko, Andrew (2000), "The Internet and other networks: utilization rates and their implications," Information Economics and Policy, Volume 12, Issue 4, December 2000, Pages 341-365.

Pupillo, Lorenzo and Giovanni Amendola (2008), “The Economics of Next Generation Access Networks and Regulatory Governance: Towards Patterns of Regulation," Communications \& Strategies, No. 69 (2008) 85-105 (available at: http://mpra.ub.uni-muenchen.de/8823/).

Reding, Viviane (2006), "From Service Competition to Infrastructure Competition: the Policy Options Now on the Table," Speech by Vivianne Reding, ECTA Conference 2006, Brussels, 16 http://www.uer.biz/CMSimages/en/BRUDOC_INFO_EN_321 tcm6-48301.pdf).

Rosston, Jeffrey and Michael Topper (2009), "An Antitrust Analysis the Case for Wireless Net Neutrality," forthcoming in Information, Economics and Policy, 2009.

Schankerman, Mark (1996), "Symmetric regulation for competitive telecommunications," Information Economics and Policy 8 (1996) 3-23.

Shakkottai, Sanjay, Theodore Rappaport, and Peter Karlsson (2003), "Cross-layer design for wireless networks," draft mimeo, June 23, 2003 (available at: http://users.ece.utexas.edu/ shakkott/Pubs/cross-layer.pdf)

Sharma, Chetan (2008), "US Wireless Data Market: Q1 2008 Update," Chetan Sharma Consulting, available at: http://www.chetansharma.com, 2008.

Sicker, Douglas and Lisa Blumensaadt (2005), "Misunderstanding the Layered Models," Journal on Telecommunications \& High Technology Law, pp. 44 - 111, Fall 2005.

Sicker, Douglas, Josh Mindel, and Cameron Cooper (1999), "The Internet Interconnection Conundrum," unpublished FCC working paper (1999).

Spiwak, Lawrence (2004), “A little intellectual honesty please...”, Phoenix Center for Advanced Legal \& Economic Public Policy Studies, 15 June 2004 (available from: http://papers.ssrn.com/sol3/papers.cfm?abstract_id=557336).

Ward, Michael and Glenn Woroch (2005), "Fixed-Mobile Telephone Subscription Substitution in the U.S.," Department of Economics Working Paper Series 05-003, University of Texas, 2005 (available at: http://www.uta.edu/faculty/mikeward/MFS3.pdf).

Werbach, Kevin (2002), "A layered model for Internet policy," Journal of Telecommunications and High Technology Law, vol 1 (2002) pages 58-64.

Whitt, Richard (2004). " A Horizontal Leap Forward: Formulating a New Communications Public Policy Framework Based on the Network Layers Model," Federal Communications Law Journal, 56 (2004) 587. 
Wu, Tim (2003), "Network Neutrality, Broadband Discrimination," Journal of Telecommunications and High Technology Law, Vol. 2, p. 141, 2003.

Wu, Tim (2007), "Wireless Carterfone," International Journal of Communication, Vol. 1, p. 389, 2007

Page 27 of 28 
Figure 1. Evolution of wired networks from silos to a common platform architecture.

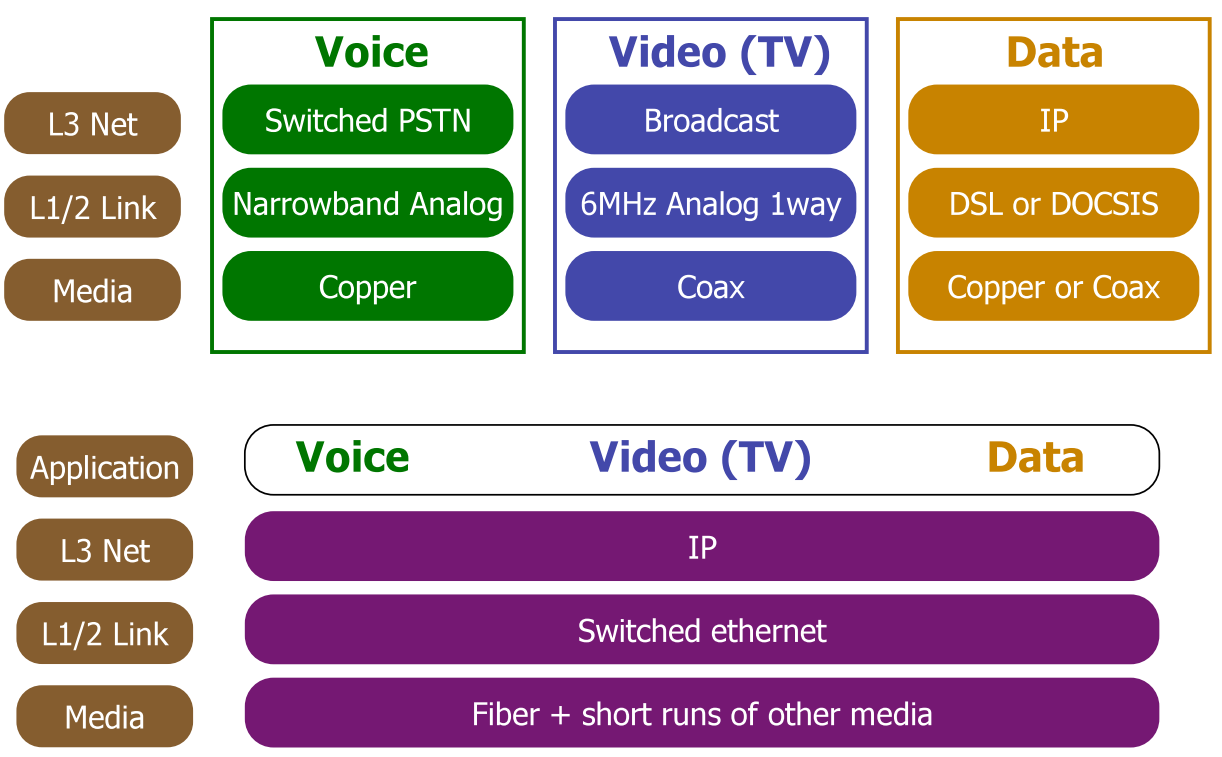

